\title{
On hamiltonian chain saturated uniform hypergraphs
}

\author{
Aneta Dudek and Andrzej Żak $\|^{\prime}$ \\ AGH University of Science and Technology, Krakow, Poland \\ received $15^{\text {th }}$ April 2010, revised $5^{\text {th }}$ September 2011, accepted 22 $2^{\text {nd }}$ November 2011.
}

We say that a hypergraph $H$ is hamiltonian chain saturated if $H$ does not contain a hamiltonian chain but by adding any new edge we create a hamiltonian chain in $H$. In this paper we ask about the smallest size of a $k$-uniform hamiltonian chain saturated hypergraph. We present a construction of a family of $k$-uniform hamiltonian chain saturated hypergraphs with $O\left(n^{k-1 / 2}\right)$ edges.

Keywords: saturated hypergraph, hamiltonian path, hamiltonian cycle, hamiltonian chain.

\section{Introduction}

Let $H$ be a $k$-uniform hypergraph on the vertex set $V(H)=\left\{v_{1}, \ldots, v_{n}\right\}$ with $n \geq k$. The set of the edges $-k$-element subsets of $V(H)-$ is denoted by $\mathcal{E}(H)$. For simplicity of notation $v_{n+x}$ with $x \geq 0$ denotes the same vertex as $v_{x}$. In [19] the authors defined the notion of a hamiltonian chain.

Definition 1 A cyclic ordering $\left(v_{1}, v_{2}, \ldots, v_{n}\right)$ of the vertex set is called a hamiltonian chain and denoted $C_{n}^{(k)}$, if and only if $\left\{v_{i}, v_{i+1}, \ldots, v_{i+k-1}\right\} \in \mathcal{E}(H)$ whenever $1 \leq i \leq n$. An ordering $\left(v_{1}, v_{2}, \ldots, v_{l}\right)$ of a subset of the vertex set is called a path and denoted $P_{l}^{(k)}$, if and only if $\left\{v_{i}, v_{i+1}, \ldots, v_{i+k-1}\right\} \in \mathcal{E}(H)$ whenever $1 \leq i \leq l-k+1$. A path $P_{n}^{(k)}$ is a hamiltonian path.

For $v \in V(H)$, let $H-v$ be the hypergraph obtained by deleting $v$ and all edges incident to $v$. We refer to this operation as removing $v$ from $H$.

Definition 2 We say that a hypergraph $H$ is hamiltonian chain saturated if $H$ does not contain a hamiltonian chain but by adding any new edge we create a hamiltonian chain in $H$.

The question investigated in this paper is the following: What is the minimum number sat $\left(n, C_{n}^{(k)}\right)$ of edges in a hamiltonian chain saturated $k$-uniform hypergraphs on $n$ vertices?

In fact, the above problem belongs to the much wider theory of saturated graphs and hypergraphs. Given a hypergraph $F$, we say that the hypergraph $H$ is $F$-saturated if $H$ has no $F$ as a subhypergraph,

\footnotetext{
$\dagger$ Email: $\{$ dudekane, zakandrz\}@agh. edu.pl The authors were partially supported by the Polish Ministry of Science and Higher Education.

1365-8050 @ 2012 Discrete Mathematics and Theoretical Computer Science (DMTCS), Nancy, France
} 
but does contain $F$ after the addition of any new edge. The minimum number of edges in an $F$-saturated hypergraph on $n$ vertices is denoted by $\operatorname{sat}(n, F)$ (the maximum number is denoted by $\operatorname{ex}(n, F)$ and the problem of determining $\operatorname{ex}(n, F)$ is the Turán's problem). There are many results on sat $(n, F)$ for graphs, including complete graphs [14], cycles [1, 2, 6, 16, 17, 20, 23], complete $s$-partite graphs [3, 7, 8, 21]. In particular, the problem considered in the paper is solved for graphs. Namely, sat $\left(n, C_{n}\right)=\left\lceil\frac{3 n}{2}\right\rceil$ (apart from a few small values of $n$ ) which follows from [5, 9, 10]. Furthermore, $\operatorname{sat}\left(n, P_{n}\right)=\left\lfloor\frac{3 n-1}{2}\right\rfloor$ for $n \geq 54$ [11, 15]. Much less is known for $k \geq 3$. Bollobás [4] generalized Erdős, Hajnal and Moons result [14] for complete $k$-uniform hypergraphs. Erdős, Füredi and Tuza [13] obtained $\operatorname{sat}(n, F)$ for some particular hypergraphs $F$ with few edges. Pikhurko [22] proved that $\operatorname{sat}(n, F)=O\left(n^{k-1}\right)$ for any fixed hypergraph $F$ (generalizing previous result for graphs by Erdős, Füredi and Tuza [13]). The same order of magnitude was conjectured for $\operatorname{sat}\left(n, C_{n}^{(k)}\right)$ [18] and $\operatorname{sat}\left(n, P_{n}^{(k)}\right)$ [12]. It is known that both $\operatorname{sat}\left(n, C_{n}^{(k)}\right)$ and $\operatorname{sat}\left(n, P_{n}^{(k)}\right)$ are at least $\left(\begin{array}{l}n \\ k\end{array}\right) /(k(n-k)+1)$, see [12]. Hence the lower bound is of order $n^{k-1}$. On the other hand in [19] a construction is given of $n$-vertex hamiltonian path saturated $k$-uniform hypergraphs with

$$
\sim\left(\frac{1}{k !}-\frac{1}{2^{k}\lceil k / 2\rceil !\lfloor k / 2\rfloor !}\right) n^{k}
$$

edges. In case when $k=3$, better constructions presented in [12] give hamiltonian path saturated 3uniform hypergraphs and hamiltonian chain saturated 3-uniform hypergraphs with $O\left(n^{5 / 2}\right)$ edges. In this paper we present a non-obvious generalization of these construction for all $k \geq 4$. As a result we obtain that

$$
\operatorname{sat}\left(n, C_{n}^{(k)}\right) \leq\left(\frac{1}{k !}+\frac{1}{(k-1) !}\right) n^{k-1 / 2}+o\left(n^{k-1 / 2}\right) .
$$

We notice also that the same bound holds for $\operatorname{sat}\left(n, P_{n}^{(k)}\right)$.

\section{Construction}

In the sequel we assume that $k$ is a fixed integer greater than or equal to 4 .

Definition 3 Let $p$ and $q$ be non-negative integers and $U_{0}, U_{1}, \ldots, U_{q}$ be pairwise disjoint sets of vertices such that $\left|U_{0}\right|=p$ and $\left|U_{i}\right| \geq 2$ for $i=1,2, \ldots, q$. Define the vertex set of the hypergraph $H$ to be $V(H)=\bigcup_{i=0}^{q} U_{i}$. Let $u_{1} \in U_{i_{1}}, \ldots, u_{k} \in U_{i_{k}}$ and $j=\min \left\{i_{1}, \ldots, i_{k}\right\}$. Then $\left\{u_{1}, \ldots, u_{k}\right\}=: E \in \mathcal{E}(H)$ if and only if $j=0$ or $\left|E \cap U_{j}\right| \geq 2$. The family of all hypergraphs obtained by this construction is denoted by $\mathcal{H}_{k}(p, q)$.

Notation The above number $j$ that is determined by the edge $E$ is denoted by $j_{E}$.

Theorem 1 Let $H \in \mathcal{H}_{k}(p, q)$ where $p, q$ are non-negative integers such that $p \geq 2$ and $q \geq \frac{k}{2}(p-1)+2$. Let $\left|U_{i}\right|=\alpha$ for $i=1, \ldots, q-1$ and $\frac{\alpha}{2}(k-1)<\left|U_{q}\right| \leq k \alpha$, where $\alpha$ is an integer satisfying $\alpha \geq 2 p(k-2)$. Then $H$ does not have a hamiltonian chain.

Proof. Suppose for a contradiction that $H$ contains a hamiltonian chain $\left(v_{1}, v_{2}, \ldots, v_{n}\right)$. By removing all vertices from $U_{0}$ the chain $\left(v_{1}, v_{2}, \ldots, v_{n}\right)$ falls to $m$ parts, $m \leq p$. Each part induces a path in $H-U_{0}$ or consists of at most $k-1$ vertices. Note that for any two adjacent edges $E$ and $E^{\prime}$ belonging to one part $j_{E}=j_{E^{\prime}}$. Indeed, $E^{\prime}$ has only one vertex which does not belong to $E$. Thus, since $\left|E \cap U_{j_{E}}\right| \geq 2, E^{\prime}$ contains a vertex from $U_{j_{E}}$. Hence, $j_{E^{\prime}} \leq j_{E}$. Due to symmetry we have also $j_{E} \leq j_{E^{\prime}}$. Therefore, every 
edge in a part has at least two vertices from some fixed set $U_{j}$. We say that the set $U_{j}$ is a dominating set for this part. Let $x_{i}$ denote the number of vertices of the $i$-th part which belong to its dominating set. Let $y_{i}$ denote the number of remaining vertices in the $i$-th part. In this part consider now consecutive disjoint $k$-tuples and an $r$-tuple at the end, $0 \leq r \leq k-1$. Let $x_{r}$ denote the number of vertices from the dominating set that belong to the $r$-tuple. Each $k$-tuple contains at least two vertices from the dominating set. Hence, the number of considered $k$-tuples does not exceed $\left\lfloor\frac{x_{i}-x_{r}}{2}\right\rfloor \leq \frac{\alpha}{2}$. Thus, if $r \leq k-2$ then the number of vertices in the $i$-th part satisfies

$$
x_{i}+y_{i} \leq \frac{\alpha}{2} k+(k-2) \text {. }
$$

If $r=k-1$ then $x_{r} \geq 1$ (because the last $k$ vertices form an edge, too). Hence, $x_{i}+y_{i} \leq\left\lfloor\frac{\alpha-1}{2}\right\rfloor k+$ $(k-1)$, so 1 is satisfied, too. Hence, $x_{i}+y_{i} \leq \frac{\alpha}{2} k+(k-2)$ or, if a part contains vertices from only one set $U_{j}, x_{i}+y_{i} \leq\left|U_{j}\right|$. In particular, $x_{i}+y_{i} \leq \frac{\alpha}{2} k+(k-2)$ for all parts except possibly one which contains only vertices from $U_{q}$ (this is because $\left|U_{q}\right|<2\left(\frac{k}{2} \alpha+(k-2)\right)$. Hence, if some part contains only vertices from $U_{q}$ then

$$
\begin{aligned}
(q-1) \alpha+\left|U_{q}\right| & =\left|U_{1}\right|+\ldots+\left|U_{q}\right|=\sum_{i=1}^{m}\left(x_{i}+y_{i}\right) \leq \sum_{i=1}^{m-1}\left(\frac{k}{2} \alpha+(k-2)\right)+\left|U_{q}\right| \\
& \leq(p-1)\left(\frac{k}{2} \alpha+(k-2)\right)+\left|U_{q}\right|, \text { hence } \\
q & \leq(p-1) \frac{k}{2}+\frac{k-2}{\alpha}(p-1)+1<(p-1) \frac{k}{2}+2, \text { a contradiction. }
\end{aligned}
$$

Otherwise,

$$
\begin{aligned}
(q-1) \alpha+\left|U_{q}\right| & =\left|U_{1}\right|+\ldots+\left|U_{q}\right|=\sum_{i=1}^{m}\left(x_{i}+y_{i}\right) \leq \sum_{i=1}^{m}\left(\frac{k}{2} \alpha+(k-2)\right) \\
& \leq p\left(\frac{k}{2} \alpha+(k-2)\right), \text { hence } \\
(q-1) \alpha & <p\left(\frac{k}{2} \alpha+(k-2)\right)-\frac{\alpha}{2}(k-1), \text { whence } \\
q & <(p-1) \frac{k}{2}+2, \text { a contradiction again. }
\end{aligned}
$$

Theorem 2 Let $t$ be a positive integer and let $H \in \mathcal{H}_{k}(2 t+1, k t+2)$. Let $\left|U_{i}\right|=\alpha$ for $i=1, \ldots, k t+1$ and $\frac{\alpha}{2}(k-1)<\left|U_{k t+2}\right| \leq(\alpha-k)(k-2)$, where $\alpha$ is an integer satisfying $\alpha \geq(4 t+2)(k-2)$. Then $H$ is hamiltonian chain saturated.

Proof. Let $p=2 t+1$ and $q=k t+2$. Hence, $q=\frac{k}{2}(p-1)+2$. Thus, by Theorem $1, H$ does not have any hamiltonian chain. We will prove that adding any new edge $E_{0}$ produces a hamiltonian chain in $H$. Let $E_{0}$ have the form $\left\{i_{1}, i_{2}, \ldots, i_{k}\right\}$ with $1 \leq i_{1}<i_{2} \leq i_{3}, \ldots, \leq i_{k} \leq q$, which means that $E_{0}$ contains a vertex from set $U_{i_{1}}$, a vertex from set $U_{i_{2}}$, and so on. Note that $E_{0}$ contains only one vertex from $U_{i_{1}}$ 
since $E_{0} \notin \mathcal{E}(H)$.

Suppose first that $i_{2} \neq q$. Thus, $E_{0}$ contains at most $k-2$ vertices from $U_{q}$. Let $j_{1}<j_{2}<\ldots<j_{q-3}$ be consecutive integers from $\{1, \ldots, q-1\} \backslash\left\{i_{1}, i_{2}\right\}$. Let $\mathcal{C}_{j_{i}}, i=1, \ldots, p-1$, be a path of the form

$$
\underbrace{*, \ldots, *}_{k-2}, j_{i}, j_{i}, \underbrace{*, \ldots, *}_{k-2}, j_{i}, j_{i}, \cdots, \underbrace{*, \ldots, *}_{k-2}, j_{i}, j_{i}, \underbrace{*, \ldots, *}_{k-2}, j_{i}, j_{i}, \underbrace{*, \ldots, *}_{k-2},\left(j_{i}\right)
$$

which means that $\mathcal{C}_{j_{i}}$ contains

- all vertices from $U_{j_{i}} \backslash E_{0}$ - in the positions denoted by $j_{i}$ (the symbol $\left(j_{i}\right)$ means that $\mathcal{C}_{j_{i}}$ may or may not contain a vertex from $U_{j_{i}} \backslash E_{0}$ in this position depending on the parity of $\left|U_{j_{i}} \backslash E_{0}\right|$ )

- some vertices from the set $\left(U_{j_{p}} \cup \ldots \cup U_{j_{q-3}}\right) \backslash E_{0}$ - in the positions denoted by $*$.

Clearly, $U_{j_{i}}$ is the dominating set for $\mathcal{C}_{j_{i}}$ (since $i<p$ ). Moreover, among every $k$ consecutive vertices there are two vertices from $U_{j_{i}}$. Thus, $\mathcal{C}_{j_{i}}$ is indeed a path. Let $z_{j_{i}}=\left|U_{j_{i}} \cap E_{0}\right|$. Note that a single $\mathcal{C}_{j_{i}}$ may contain $\left\lfloor\frac{\alpha-z_{j_{i}}}{2}\right\rfloor k+(k-2)$ vertices. Thus, the paths $\mathcal{C}_{j_{1}}, \ldots, \mathcal{C}_{j_{p-1}}$ together may contain $(q-3) \alpha$ vertices. Indeed,

$$
\begin{aligned}
\sum_{i=1}^{p-1}\left(\left\lfloor\frac{\alpha-z_{j_{i}}}{2}\right\rfloor k+(k-2)\right) & \geq \sum_{i=1}^{p-1}\left(\frac{\alpha-z_{j_{i}}-1}{2} k+(k-2)\right) \\
& =\frac{\alpha}{2} k(p-1)+(p-1)(k-2)-\sum_{i=1}^{p-1} \frac{z_{j_{i}}+1}{2} k \\
& =\frac{\alpha}{2} k(p-1)+(p-1)(k-2)-\frac{k}{2}\left(\sum_{i=1}^{p-1} z_{j_{i}}+\sum_{i=1}^{p-1} 1\right) \\
& \geq \frac{\alpha}{2} k(p-1)+(p-1)(k-2)-\frac{k}{2}(k-2+p-1) \\
& \geq \frac{\alpha}{2} k(p-1)-\alpha=(q-3) \alpha, \text { because } \alpha \geq \frac{k}{2}(k-2) .
\end{aligned}
$$

On the other hand, after removing any number of vertices outside the set $U_{j_{i}}$ from a $\mathcal{C}_{j_{i}}$ we still have a path. Indeed, by removing some vertices from positions denoted by $*$ we do not spoil the property, that among every $k$ consecutive vertices there are at least two vertices from $U_{j_{i}}$. Therefore we can construct $p-1$ paths $\mathcal{C}_{j_{1}}^{\prime}, \ldots, \mathcal{C}_{j_{p-1}}^{\prime}$ which together contain all vertices from $\left(U_{j_{1}} \cup \ldots \cup U_{j_{q-3}}\right) \backslash E_{0}$ (and do not contain any other vertex). Finally, let $\mathcal{C}_{i_{1}}$ have the form

$$
\underbrace{q, \ldots, q}_{k-2}, i_{1}, i_{1}, \underbrace{q, \ldots, q}_{k-2}, i_{1}, i_{1}, \cdots, \underbrace{q, \ldots, q}_{k-2}, i_{1}, \underbrace{i_{1}, \overbrace{i_{3}, \ldots, i_{k}}^{k-2}, i_{2}}_{E_{0}} i_{2} \underbrace{q, \ldots, q}_{k-2}, \cdots, i_{2}, i_{2}, \underbrace{q, \ldots, q}_{k-2} .
$$

Note that

$$
\begin{aligned}
\left\lfloor\frac{\left|U_{i_{1}} \cup U_{i_{2}}\right|-(k-2)}{2}\right\rfloor & \geq \frac{2 \alpha-k+1}{2}=\alpha-k+k / 2+1 / 2>\alpha-k+2 \\
& =\frac{(\alpha-k)(k-2)}{k-2}+2>\left\lceil\frac{\left|U_{q}\right|}{k-2}\right\rceil .
\end{aligned}
$$


Hence, we are able to insert all vertices from $U_{q}$ to $\mathcal{C}_{i_{1}}$. Moreover, by adding at the beginning (or at the end) of $\mathcal{C}_{i_{1}}$ any number of vertices from $U_{i_{1}} \backslash E_{0}$ (from $U_{i_{2}} \backslash E_{0}$ ) we still have a path. Hence, we can construct a path $\mathcal{C}^{\prime}{ }_{i_{1}}$ which contains all vertices from $U_{i_{1}} \cup U_{i_{2}} \cup U_{q} \cup E_{0}$. It is easy to see now that

$$
\left(\mathcal{C}_{j_{1}}^{\prime}, 0, \mathcal{C}_{j_{2}}^{\prime}, 0, \cdots, 0, \mathcal{C}_{j_{p-1}}^{\prime}, 0, \mathcal{C}_{i_{1}}^{\prime}, 0\right)
$$

(here symbols 0 denote distinct vertices from $U_{0}$ ) is a hamiltonian chain in $H$.

Suppose now that $i_{2}=q$, whence $E_{0}$ is of the form $\left\{i_{1}, q, \ldots, q\right\}$. Let $j_{1}<j_{2} \ldots<j_{q-2}$ be consecutive integers from $\{1, \ldots, q-1\} \backslash\left\{i_{1}\right\}$. We construct paths $\mathcal{C}_{j_{1}}, \ldots, \mathcal{C}_{j_{p-1}}$ in a similar way as previously. This time the paths $\mathcal{C}_{j_{i}}$ may contain $(q-2) \alpha$ vertices. This can be verified by similar computations as previously, see (2), with one difference that now $z_{j_{i}}=0$ for each $i=1, \ldots, p-1$. Recall, that after removing any number of vertices outside the set $U_{j_{i}}$ from a $\mathcal{C}_{j_{i}}$ we still have a path. Therefore, we can construct $p-1$ paths $\mathcal{C}_{j_{1}}^{\prime \prime}, \ldots, \mathcal{C}_{j_{p-1}}^{\prime \prime}$ which together contain all vertices from $U_{j_{1}} \cup \ldots \cup U_{j_{q-2}}$ (and do not contain any other vertex). Now let $\mathcal{C}_{i_{1}}^{\prime \prime}$ have the form

$$
i_{1}, i_{1}, \ldots, i_{1}, \underbrace{i_{1}, q, q, \ldots, q}_{E_{0}}, q, \ldots, q, q
$$

and contain all vertices from $U_{i_{1}} \cup U_{q}$. Therefore,

$$
\left(\mathcal{C}_{j_{1}}^{\prime \prime}, 0, \mathcal{C}_{j_{2}}^{\prime \prime}, 0, \cdots, 0, \mathcal{C}_{j_{p-1}}^{\prime \prime}, 0, \mathcal{C}_{i_{1}}^{\prime \prime}, 0\right)
$$

is a hamiltonian chain in $H$.

\section{Main result}

Theorem 3 For each $n \geq k^{4}+8 k^{3}+16 k^{2}$, there exists a $k$-uniform, hamiltonian chain saturated hypergraph with fewer than $\left(\frac{1}{k !}+\frac{1}{(k-1) !}\right) n^{k-1 / 2}+o\left(n^{k-1 / 2}\right)$ edges.

Proof. Let $t_{0}:=\left\lfloor\frac{\sqrt{n}}{2 k}\right\rfloor-1$ and $\alpha:=\left\lfloor\frac{n-2 t_{0}-2}{k t_{0}+1 / 2+k / 2}\right\rfloor$. Thus, it is easy to check that $\alpha \geq\left(4 t_{0}+2\right) k>$ $\left(4 t_{0}+2\right)(k-2)$. Let $H \in \mathcal{H}_{k}\left(2 t_{0}+1, k t_{0}+2\right)$ with $\left|U_{i}\right|=\alpha$ for $i=1, \ldots, k t_{0}+1$. Then

$$
\begin{aligned}
\frac{n-2 t_{0}-1}{k t_{0}+1 / 2+k / 2}-1 & \leq \alpha<\frac{n-2 t_{0}-1}{k t_{0}+1 / 2+k / 2}, \text { whence } \\
\left(k t_{0}+1\right) \alpha+\frac{\alpha}{2}(k-1) & <n-2 t_{0}-1 \leq\left(k t_{0}+1\right) \alpha+\frac{\alpha}{2}(k-1)+\left(k t_{0}+k / 2+1 / 2\right) .
\end{aligned}
$$

Thus, since $n-2 t_{0}-1=\left(k t_{0}+1\right) \alpha+\left|U_{k t_{0}+2}\right|$, we have

$$
\frac{\alpha}{2}(k-1)<\left|U_{k t_{0}+2}\right| \leq \frac{\alpha}{2}(k-1)+k t_{0}+k / 2+1 / 2<(\alpha-k)(k-2)
$$

for $k \geq 4, \alpha \geq\left(4 t_{0}+2\right) k$ and $t_{0} \geq k / 2$. Therefore, by Theorem $2, H$ is a hamiltonian chain saturated hypergraph. 
Moreover,

$$
\begin{aligned}
|\mathcal{E}(H)| & \leq\left|U_{0}\right|\left(\begin{array}{l}
n-1 \\
k-1
\end{array}\right)+\sum_{i=1}^{k t_{0}+1}\left(\begin{array}{l}
\alpha \\
2
\end{array}\right)\left(\begin{array}{c}
n-2 t_{0}-1-(i-1) \alpha \\
k-2
\end{array}\right)+\left(\begin{array}{c}
\left|U_{k t_{0}+2}\right| \\
k
\end{array}\right) \\
& \leq\left(2 t_{0}+1\right) \frac{n^{k-1}}{(k-1) !}+\frac{\alpha^{2}}{2} \sum_{i=1}^{k t_{0}+1} \frac{(n-(i-1) \alpha)^{k-2}}{(k-2) !}+\frac{k^{k} \alpha^{k}}{k !}
\end{aligned}
$$

Comparing the sum of the areas of rectangles $R_{i}, i=1, \ldots, k t_{0}+1$, with sides $a_{i}=\alpha$ and $b_{i}=$ $(n-(i-1) \alpha)^{k-2}$ with the area of the figure limited by the $x$-axis and the graph of the function $y=x^{k-2}$, $0 \leq x \leq n+\alpha$, we obtain that

$$
\begin{aligned}
& \sum_{i=1}^{k t_{0}+1} \alpha(n-(i-1) \alpha)^{k-2} \leq \int_{0}^{n+\alpha} x^{k-2} d x, \text { hence }, \\
& \sum_{i=1}^{k t_{0}+1}(n-(i-1) \alpha)^{k-2} \leq \frac{(n+\alpha)^{k-1}}{\alpha(k-1)}
\end{aligned}
$$

Therefore,

$$
\begin{aligned}
|\mathcal{E}(H)| & \leq\left(2 t_{0}+1\right) \frac{n^{k-1}}{(k-1) !}+\frac{\alpha(n+\alpha)^{k-1}}{2(k-1) !}+\frac{k^{k} \alpha^{k}}{k !} \\
& =\left(\frac{1}{k !}+\frac{1}{(k-1) !}\right) n^{k-1 / 2}+o\left(n^{k-1 / 2}\right),
\end{aligned}
$$

because $t_{0} \approx \frac{\sqrt{n}}{2 k}$ and $\alpha \approx 2 \sqrt{n}$.

\section{Concluding remarks}

We have constructed a family of $k$-uniform hamiltonian chain saturated hypergraphs. The main result is Theorem 3 , which gives the hypergraphs with the smallest known number of edges. Unfortunately, since the lower bound in [12] is smaller than the number of edges in our construction by a factor $n^{1 / 2}$ the order of magnitude for $\operatorname{sat}\left(C_{n}^{(k)}\right)$ remains unknown.

Note that our construction cannot be significantly improved by taking another $t_{0}$. Indeed, if we take $t_{0}$ of order greater than $n^{1 / 2}$ then, the first component of 44 is of order greater than $n^{k-1 / 2}$. Otherwise, if $t_{0}$ is of order less than $n^{1 / 2}$, then $\alpha$ is of order greater than $n^{1 / 2}$, whence the second component of (4) is of order greater than $n^{k-1 / 2}$. On the other hand, the coefficient $\frac{1}{2 k}$ is chosen in order to minimize (4) and satisfy all necessary conditions that the numbers $p, q, \alpha$ must have (some slight improvements are still possible, however).

We observe that the same bounds can be obtained in case when we consider hamiltonian paths. The upper bound can be realized by a hypergraph $H \in \mathcal{H}_{k}\left(2 t_{0}, k t_{0}+2\right)$ with $t_{0}$ and $\alpha$ being the same as in Theorem 3

Theorem 4 For each $n \geq k^{4}+8 k^{3}+16 k^{2}$, there exists a $k$-uniform, hamiltonian path saturated hypergraph with less than $\left(\frac{1}{k !}+\frac{1}{(k-1) !}\right) n^{k-1 / 2}+o\left(n^{k-1 / 2}\right)$ edges. 


\section{References}

[1] Y. Ashkenazi, $C_{3}$ saturated graphs, Discrete Math. 297 (2005), 152-158.

[2] C. A. Barefoot, L. H. Clark, R. C. Entringer, T. D. Porter, L. A. Székely and Zs. Tuza, Cyclesaturated graphs of minimum size. Selected papers in honour of Paul Erdős on the occasion of his 80th birthday (Keszthely, 1993), Discrete Math. 150 (1996), 31-48.

[3] T. Bohman, M.Fonoberova and O. Pikhurko, The saturation function of complete partite graphs, Journal of Combinatorics 1 (2010), 149-170.

[4] B. Bollobás, On generalized graphs, Acta Math. Acad. Sci. Hungar 16 (1965), 447-452.

[5] J. A. Bondy, Variations on the hamiltonian theme, Canad. Math. Bull. 15 (1972) 57-62.

[6] Y. Chen, Minimum $C_{5}$-saturated graphs, J. Graph Theory 61 (2009), 111-126.

[7] Ya-Chen Chen, Minimum $K_{2,3}$-saturated graphs, submitted.

[8] G. Chen, R. Faudree and R. Gould, Saturation numbers of books, Electron. J. Combin. 15 (2008), Research Paper 118, 12 pp.

[9] L. H. Clark, R. C. Entringer, Smallest maximally nonhamiltonian graphs, Period. Math. Hung. 14 (1983), 57-68.

[10] L. H. Clark, R. C. Entringer, H. D. Shapiro, Smallest maximally nonhamiltonian graphs II, Graphs and Combin. 8 (1992) 225-231.

[11] A. Dudek, G. Y. Katona, A. P. Wojda, Hamiltonian path saturated graphs with small size, Discrete Appl. Math. 154 (2006) 1372-1379.

[12] A. Dudek, G. Y. Katona, A. Żak, Hamilton-chain saturated hypergraphs, Discrete Math. 310 (2010) 1172-1176.

[13] P. Erdôs, Z. Füredi and Zs. Tuza, Saturated $r$-uniform hypergraphs, Discrete Math. 98 (1991), 95104.

[14] P. Erdős, A. Hajnal and J. W. Moon, A problem in graph theory, Amer. Math. Monthly 71 (1964), 1107-1110.

[15] M. Frick, J. Singleton, Lower bound for the size of maximal nontraceable graphs, Electronic J. Combin. 12 (2005) R32.

[16] Z. Füredi and Younjin Kim, Cycle-saturated graphs with minimum number of edges, submitted.

[17] R. Gould, T. Łuczak and J. Schmitt, Constructive upper bounds for cycle-saturated graphs of minimum size,Electron. J. Combin. 13 (2006), Research Paper 29, 19 pp.

[18] G.Y. Katona, Hamiltonian chains in hypergraphs, DMTCS proc. AE, 2005, 345-350.

[19] G.Y. Katona, H. Kierstead, Hamiltonian chains in hypergraphs, J. Graph Theory 30 (1999) 205-212. 
[20] L. T. Ollmann, $K_{2,2}$ saturated graphs with a minimal number of edges, Proceedings of the Third Southeastern Conference on Combinatorics, Graph Theory, and Computing (Florida Atlantic Univ., Boca Raton, Fla., 1972), Florida Atlantic Univ., Boca Raton, Fla., 1972, pp. 367-392.

[21] O. Pikhurko, Results and open problems on minimum saturated hypergraphs, Ars Combin. 72 (2004), 111-127.

[22] O. Pikhurko, The minimum size of saturated hypergraphs, Combin. Probab. Comput. 8 (1999), 483492.

[23] Zs. Tuza, $C_{4}$-saturated graphs of minimum size, Acta Univ. Carolin. Math. Phys. 30 (1989), 161167.

[24] L. Xiaohui, J. Wenzhou, Z. Chengxue, Y. Yuansheng, On smallest maximally nonhamiltonian graphs, Ars Combin. 45 (1997) 263-270. 